\title{
Modernización curricular en la Universidad Pontificia Bolivariana: una aplicación a la seccional Palmira
}

\section{Curricular modernization in Universidad Pontificia Bolivariana: An application to its siege in Palmira, Valle del Cauca, Colombia.}

María Adelaida Upegui Córdoba

Yovany Ospina Nieto

Edwin Javier Ortega Zúñiga

Universidad Pontificia Bolivariana, sede Palmira. Grupo de Investigación en Pedagogía y Desarrollo Humano.

Autor para correspondencia:yovany.ospina@ upb.edu.co

\section{Resumen}

En este artículo se describe el proyecto investigativo titulado "Modernización curricular en la Universidad Pontificia Bolivariana, Seccional Palmira", cuyo propósito fue diseñar un programa de modernización curricular para integrar conscientemente -en su comunidad académico y administrativa- la carta de navegación Proyecto Institucional (PI) y el Modelo Pedagógico Integrado (MPI) que rige a la Universidad Pontificia Bolivariana, Seccional Palmira. Se tuvieron en cuentas estas subcategorías: formación, investigación y proyección social, con las que se pretendía hacer un estudio juicioso que brindara soluciones efectivas. Las primeras actividades que se realizaron fueron: a) Análisis del proceso de formación de los programas de la universidad, b) Planeación de actividades, c) Ejecución de actividades que permiten la modernización curricular. Después, se llevó a cabo: a) un Diplomado en Pedagogía, b) la Sistematización de las experiencias y c) Las asesorías a los programas académicos, para la transformación curricular.

Palabras clave: modernización curricular, formación, investigación, proyección social, Identidad Académica.

\section{Abstract}

In this article, is described the research project titled "Curriculum modernization in the Pontifical 
Bolivarian University, Sectional Palmira", whose purpose was design a curriculum modernization program to integrate consciously - in their academic and administrative community- the navigation chart Institutional Project (PI) and Integrated Teaching Model (MPI) that govern in the Pontifical Bolivarian University, Sectional Palmira. They were taken into account these subcategories: training, investigation and social projection, with these has pretended make a wise study that provide effective solutions. The first activities that it was performed, were: a) Analysis the training's process of the University's programs, b) Activities planning, c) Execution of activities that allow the curriculum modernization. Then, it carried out: a) a Diploma in Pedagogy, b) the experiences' Systematization and c) the advices to the academic programs, for the curriculum transformation.

Keywords: curriculum modernization, training, research and outreach, academic identity.

\section{Introducción}

El proyecto de investigación de Modernización Curricular en la Universidad Pontificia Bolivariana, Seccional Palmira surge a raíz de la evaluación realizada por los pares internos de Medellín. En dicha evaluación, se evidenciaron debilidades por parte de los docentes y los directivos docentes en el proceso formativo y en la falta de conocimiento del Proyecto Institucional (PI) y del Modelo Pedagógico Integrado (MPI) de la Universidad. En otras palabras, desde la práctica pedagógica, no se está cumpliendo con la función esencial de la universidad: brindar formación, investigación y proyección social. Y este compromiso institucional está descrito en el Proyecto Institucional (2004, p. 12), cuya misión es: "la búsqueda de la verdad en los procesos de docencia, investigación y proyección social". Toda vez que estas acciones son las que permiten que la universidad viabilice la formación profesional integral; muy bien orientada en el Modelo Pedagógico Integrado (2009, p. 5). Esta formación se fundamenta en "la formación de capacidades y competencias axiológicas, cognitivas y procedimentales", desde una formación investigativa, basada en el aprender a aprender, para que los estudiantes logren dar respuesta a las necesidades de la sociedad y del sector productivo.
Partiendo de esta necesidad, se diseñó la propuesta de investigación, para presentarla al Comité de Investigación para su aprobación. La propuesta está centrada en la categoría de Modernización Curricular, que, según Correa (2015, p. 2), es la que permite hacer el proceso de reflexión permanente, crítica y sistemática, que posibilita la interpretación, explicación y anticipación de los procesos de formación profesional, y que garantiza las pertinencias sociales, científicas y culturales a la formación integral de sus egresados, igualmente, a los requerimientos del complejo mundo global y a la internacionalización de la educación superior.

Sería muy bueno que la Seccional Palmira se apropie de esta interesante perspectiva, para que le dé cumplimiento al proceso de acreditación de alta calidad.

Asimismo, en el proyecto confluyen otras subcategorías, a saber: formación, investigación y proyección social, las cuales permitirán hacer un estudio juicioso que brinde soluciones a la problemática que plantea el proyecto de investigación. Upegui manifiesta que "la docencia es una tarea de transferencia y extensión, en la cual se evidencia un talento muy particular en el docente" (2010, p. 81); y que "Con ella, se hace alusión a la más insigne función de la universidad contemporánea, que se ha erigido como su razón de ser: la producción del conocimiento" (2010, p. 84).

La modernización curricular es, hoy por hoy, uno de los temas más abiertos al debate de las ciencias sociales, por las implicaciones que tiene en los procesos de la educación superior. A través de esta, en efecto, se pueden revisar las debilidades y fortalezas que desarrolla el proceso de ejecución de la formación. Así lo confirma la Universidad del Norte de Barranquilla: "El Programa de Modernización Curricular requiere acciones, por una parte, que faciliten su aseguramiento en función de la organización, sistematización y transferencia de datos $y$, de otra parte, que permitan verificar y evaluar la calidad de los procesos" (2002, p.5). Por esto, el proyecto de investigación aprovecha el espacio del I Encuentro de Semilleros que realiza la UPB, Seccional Palmira, para presentar a la comunidad educativa sus avances, a través de una programación de ponencias. 


\section{Descripción del proyecto}

En el primer semestre del 2015, el proyecto realizó: 1) Análisis del proceso de formación de los programas de la Universidad; 2) Planeación de actividades; 3) Ejecución de actividades que permiten la modernización curricular. La primera actividad fue la reunión del equipo de investigadores, en la que se ejecutó el plan de trabajo y se dejaron consignadas las actividades, las metas, las fechas estimadas de realización, los responsables asignados a la actividad y los resultados esperados.

\section{Análisis del proceso de formación de los programas de la universidad}

El análisis del proceso de formación de los programas de la Seccional está basado en el Sistema Nacional de Acreditación, pues es el que permite aplicar un "conjunto de políticas, estrategias, procesos y organismos, cuyo objetivo fundamental es garantizar a la sociedad que las instituciones de educación superior que hacen parte del sistema cumplen con los más altos requisitos de calidad y que realizan sus propósitos y objetivos" (artículo 53 de la Ley 30 de 1992, p.1)

Con base en lo anterior, el proyecto de Modernización Curricular ha generado estrategias en la Seccional Palmira que permiten fortalecer la calidad académica, con el propósito de hacer procesos de autoevaluación que garanticen el aseguramiento de la calidad académica. Una de estas estrategias fue generar un análisis en el proceso formativo de los programas de la universidad. Por ello, se diseñó una encuesta que midiera la ejecución de la formación profesional en el contexto universitario.

Esto, con la intención de establecer la estructura, organización, identidad y relaciones de los actores pertenecientes a la Seccional. A la fecha, ya se analizó la encuesta y se viene adelantando un artículo de investigación para presentar a una revista indexada. También se diseñaron instrumentos de evaluaciones diagnósticas y de satisfacción de los programas de cualificación docente.

\section{Planeación de actividades que aseguren el proyecto de la modernización curricular innovadora}

Se han tenido en cuenta los lineamientos generales del Plan de Desarrollo Institucional de la UPB, Seccional Palmira, para el período 20142019. En este documento, se resalta que "Sólo una universidad que demuestra su excelencia día a día y que es capaz de construir alternativas será vista como una entidad relevante y necesaria para la sociedad" (2014, p. 9). Asimismo, el Plan de Desarrollo Institucional de la UPB, Seccional de Montería, para el período 2014-2017, explica que: "Una universidad a nivel administrativo y académico aplica estrategias que permitan hacer más efectiva la comunicación de sus lineamientos y políticas, lo cual la llevará a avanzar con éxito hacia la institución que se proyecta ser" (2014, p. 6). A partir de estas perspectivas, la Seccional Palmira, en su proyecto de investigación de modernización curricular, viene realizando actividades que garanticen una modernización innovadora.

A continuación, se presentan algunas actividades que viene adelantando el proyecto de investigación:

- Diseño e implementación de Diplomado en Pedagogía innovador, en el primer semestre 2015. Este diplomado facilitará el mejoramiento de los procesos de cualificación pedagógica e investigativa; es decir, el interés de generar estrategias que hagan más efectiva la comunicación asertiva de los docentes y administrativos docentes, en lo relacionado con los lineamientos y políticas institucionales. Igualmente, busca propiciar un espacio de continua reflexión y concienciación en el proceso pedagógico que se vive en el aula. Todo esto, con la intención de que la práctica pedagógica cumpla con la misión de la universidad y que ésta permita el impacto social que busca la visión institucional.

- Formulación de la propuesta de sistematización de experiencia del Instituto Familia yVida, desde los años 2007 al 2014, con dos propósitos: a) conocer las experiencias investigativas que ha desarrollado la universidad, a través del Instituto y b) determinar si la investigación que ha realizado 
está cumpliendo con la misión y la visión trazada en el Proyecto Educativo Institucional.

- Planeación de asesorías pedagógicas para los programas de formación de Derecho y Administración.

\section{Ejecución de actividades de modernización curricular}

Por medio de la modernización curricular de la Seccional, se han puesto en práctica los principios que recomienda Alzate (2006, p. 3), los cuales conducen hacia el "deber-ser" del proceso investigativo. De esta manera, se podrán potencializar las metas por alcanzar, y se conseguirá una especie de "llegara-ser", consecuente-coherente; y no una disposición absoluta, o punto de partida. En síntesis, se ha logrado lo siguiente:

a) Ejecución del Diplomado en Pedagogía: se inició con 18 docentes de distintos programas de la universidad, y solo culminaron satisfactoriamente 16 profesores. Su proceso fue innovador porque se logró hacer investigación y producir conocimiento: Carta Descriptiva, Proyecto formativo integrado, Proyecto de vida docente.

En el proceso de cualificación del diplomado, se conoció que los docentes que participaron no tenían estudios de maestría, lo que dificultó el proceso de cualificación de la investigación. No obstante, se logró guiar y acompañar adecuadamente a los profesores, para conseguir las estrategias de aprendizaje esperadas.

Cuando se aplicó la evaluación diagnóstica -para identificar si los profesores cumplían con la función de formar, investigar y hacer trabajo de extensión- los resultados arrojaron que están dedicados -en su mayoría- a la formación, y que han dejado de lado las funciones de investigación y de extensión.

Considerando lo anterior, el grupo de investigación de Modernización Curricular le propuso a la Dirección de Docencia que, después de tener la prueba piloto de los 16 profesores con cualificación pedagógica, se les pueda seguir acompañando, con seguimientos pedagógicos para lograr que apliquen, en la práctica académica, el conocimiento adquirido.

b) Sistematización de las experiencias: los proyectos adelantados en el Instituto Familia y Vida (IFV), se caracterizaron, de acuerdo con su tipo y se direccionaron a las correspondientes líneas de investigación, adscritas a la Coordinación de Investigación (líneas de Investigación Formativa, Proceso de Gestión de Proyectos). También, se recogieron las experiencias significativas que contribuyen con la proyección social de la Universidad Pontificia Bolivariana, Seccional Palmira. Esto se hizo con base en el concepto de sistematización de experiencias, que alude al ejercicio de producción de conocimiento crítico desde las prácticas; aunque en muchas ocasiones sólo se entiende por mera recopilación de datos, o como la narración de eventos. Por ende, es necesario comprender la sistematización, cuando se realiza con el rigor que requiere un proceso investigativo. Al respecto, Isla (2011, p.8) señala que "la sistematización como modalidad de la Investigación Acción facilita incorporar al sujeto como ente activo del proceso indagatorio en sus múltiples interrelaciones, expresiones y dimensiones desde la práctica histórica social concreta". De ahí que se pueda inferir por qué este concepto ha sido acogido por la educación superior y ha propiciado que a su alrededor se tejan interesantes puntos de reflexión en torno a su identidad específica.

El proceso de sistematización de experiencias cuenta con una bitácora y unas fases de ejecución. En la primera, se realizaron las siguientes actividades:

1. Conformación del equipo para la sistematización de experiencias del Instituto Familia yVida.

2. Determinación de los objetivos para la sistematización.

3. Definición de los lineamientos para la sistematización de las experiencias.

4. Socialización, con los líderes de proyectos, de los cuatro momentos de sistematización.

5. Lectura y clasificación de los documentos del IFV, desde hace siete años (2007).

6. Presentación de los avances de la sistematización en las reuniones de Coordinación de Investigación.

El desarrollo de esta fase se realizó en el tiempo programado, pues sólo dependía de la líder del proyecto. 
En la segunda fase, se van recopilar los proyectos y se va a tener en cuenta la participación de las personas que los han liderado en el IFV, por su aporte en la sistematización. Se ha dificultado cumplir con los tiempos, de acuerdo con lo programado en la bitácora, pues se necesita coordinar entrevistas con diferentes actores. Además, la búsqueda de información de los proyectos ha ocasionado atrasos en la planeación; en ocasiones, las personas no ven la importancia de la investigación más allá del proceso; la ven como mera digitación de la información. Para esta fase, es necesario construir la línea de tiempo de la experiencia, y determinar sus puntos más relevantes:

1. Los actores que participaron y sus roles en el proceso.

2. La intencionalidad que animó la experiencia.

3. Las fortalezas y dificultades que se presentaron en el ejercicio.

Cabe aclarar que, para poder consolidar la digitación de los proyectos, se diseñó una plantilla, en la que es fácil presentar, de manera general, los 67 proyectos que se encontraron en el IFV. Asimismo, se consolidó -en el formato enviado por la Coordinación de Gestión de Proyectos- la lista de los proyectos encontrados en el IFV: 50 Proyectos de Práctica, 4 Proyecto de Diplomado, 13 Proyectos Productivos.

c) Las asesorías a los programas académicos, para la transformación curricular. Estas consistían:

1. Se acompañó al programa de Derecho, en los ajustes de modernización curricular que debían enviar a la Dirección de Medellín.

2. Al programa de Administración, se le brindó el acompañamiento pedagógico en la revisión de la malla curricular y en la reunión con el director del Programa de Administración de Medellín.

3. Se diseñaron 5 diplomados, en actualizaciones de conocimiento psicopedagógico, para ser presentados ante el Comité de la Secretaría de Educación, los cuales fueron aprobados para ser ofertados a los profesores adscritos a la Secretaría.

\section{Conclusiones}

A partir de lo analizado, es posible, a manera de reflexión, concluir lo siguiente:
- El proyecto de Investigación de Modernización Curricular permitirá que la comunidad académico-administrativa valore y examine la carta de navegación (PEI) y el Modelo Pedagógico que rige a la Universidad Pontificia Bolivariana, Seccional Palmira.

- Asimismo, permitirá hacer un proceso de reflexión permanente, crítico y sistemático, que posibilite la interpretación, la explicación y la anticipación de los procesos de formación profesional; así, se podrá garantizar pertinencia social, científica y cultural a la formación integral de sus estudiantes y egresados, y los requerimientos del complejo mundo global y de la internacionalización de la educación superior.

- El proyecto de Investigación de Modernización Curricular basa sus principios en la orientación del currículo, centrado en el aprendizaje y en la enseñanza. De esta manera, los docentes estarán aptos para cambiar paradigmas; es decir, para dejar prácticas magistrales, y acoger nuevas formas de actuación frente a los alumnos, desde la acción y la orientación; asumiendo su papel como propiciador de aprendizajes significativos.

- El proyecto fortalece el aseguramiento de la calidad académica. Centra su atención en los docentes y los administrativos docentes, para que cumplan con la función esencial de la universidad: formar, investigar y hacer proyección social. Gracias a la formación, por medio de proyectos integrados, que les permitirá ser activos en su acompañamiento académico.

- El proyecto de investigación viabilizará la modernización curricular en la Seccional. En otras palabras, habrá evidencias tangibles para el Consejo Nacional de Acreditación (CNA), propias de una institución acreditada en alta calidad.

- Por último, este proyecto permitirá sistematizar las experiencias de investigación, lo cual, a su vez, fortalecerá las líneas y viabilizará la proyección social, desde la formación interdisciplinaria que asegura la formación integral.

\section{Referencias}

Alzate, M. (2006). Pensando el currículo. Comité de Transformación Curricular, Facultad de Ciencias Exactas y Naturales, Universidad de Antioquia (Eds.). Medellín, Colombia.125 p. 
Comité de Modernización Curricular. (2002). Proyecto de Modernización Curricular. Universidad del Norte (Eds.). Barranquilla, Colombia. $134 \mathrm{p}$.

Consejo Nacional de Acreditación. (2015). Sistema Nacional de Acreditación en Colombia. http:// www.cna.gov.co/1741/article-186365.html.

Correa, J. (2015). Modernización Curricular. Pamplona: Universidad de Pamplona (eds.). Bucaramanga, Santander-Colombia. 165 p.

Departamento de planeación. (2014). Plan de desarrollo institucional 2014. Universidad Pontificia Bolivariana, Seccional Palmira (Eds.). Palmira, Valle-Colombia. $122 \mathrm{p}$.

Departamento de planeación. (2014). Plan de Desarrollo Institucional 2014-2017. Universidad Pontificia Bolivariana Seccional de Montería (Eds.). Montería-Córdoba, Colombia. 89 p.

Isla, M. (2011). Sistematización de Experiencias. Alternativa Investigativa Participativa de las Prácticas Culturales. Centro Nacional de Superación para la Cultura (Eds.). La Habana, Cuba. $74 \mathrm{p}$.

Modelo Pedagógico Integrado. (2009). Universidad Pontificia Bolivariana. Medellín: Editorial UPB. 5 p.Proyecto Institucional. (2004). Universidad Pontificia Bolivariana. Editorial Prensa Libre. Medellín-Antioquia, Colombia. 34 p.

Upegui, M. (2010). Identidad Académica en los Profesores de Educación Superior. Editorial USC. Cali-Valle, Colombia. 36p. 